\title{
EQUICONTINUITY OF MINIMAL SETS FOR AMENABLE GROUP ACTIONS ON DENDRITES
}

\author{
ENHUI SHI \& XIANGDONG YE
}

\begin{abstract}
We show that if $G$ is an amenable group acting on a dendrite $X$, then the restriction of $G$ to any minimal set $K$ is equicontinuous, and $K$ is either finite or homeomorphic to the Cantor set.
\end{abstract}

\section{INTRODUCTION}

It is well known that every continuous action of a topological group $G$ on a compact metric space $X$ must have a minimal set $K$. A natural question is to ask what can be said about the topology of $K$, and the dynamics of the subsystem $(K, G)$. The answer to this question certainly depends on the topology of $X$ and involves the algebraic structure of $G$. We assume throughout that groups are topological groups, and that the actions are continuous.

In the case of an orientation-preserving group action on the circle $\mathbb{S}^{1}$, the topology of minimal sets and the dynamics on them are well understood. In fact, for any action of a topological group $G$ on $\mathbb{S}^{1}$, the minimal set $K$ can only be a finite set, a Cantor set, or the whole circle (see, for example, [13]). The interaction between the topology of $K$ and the algebraic structure of $G$ arises as follows.

- If $K$ is a Cantor set, then $(K, G)$ is semi-conjugate to a minimal action of $G$ on $\mathbb{S}^{1}$.

- If $K=\mathbb{S}^{1}$, then $(K, G)$ is either equicontinuous, or $(K, G)$ is $\varepsilon$-strongly proximal for some $\varepsilon>0$, and $G$ contains a free non-commutative subgroup (so, in particular, $G$ cannot be amenable; see [8]).

The classes of minimal group actions on the circle up to topological conjugacy have been classified by Ghys using bounded Euler class (see [4, 5]).

Recently, there has been considerable progress in the study of group actions on dendrites. Minimal group actions on dendrites appear naturally in the theory of 3-dimensional hyperbolic geometry (see, for example, [2, 10]). Shi proved that every minimal group action on a dendrite is strongly proximal, and the acting group cannot be amenable (see [15, 16]). Based on the results obtained by Marzougui and Naghmouchi in [9], Shi and Ye showed that an amenable group action on a dendrite always has a minimal set consisting of 1 or 2 points (see [17]), which is also implied by the work of Malyutin and DuchesneMonod (see [7, 3]). For group actions on dendrites with no finite orbits, Glasner and Megrelishvili showed the extreme proximality of minimal subsystems and the strong proximality of the whole system; for amenable group actions on dendrites, they showed that every infinite minimal subsystem is almost automorphic (see [6]). For $\mathbb{Z}$ actions on dendrites, Naghmouchi proved that every minimal set is either finite or an adding machine (see [12]).

2010 Mathematics Subject Classification. 54H20, 37B25, 37B05, 37B40.

Key words and phrases. Equicontinuity, amenable group, minimal sets. 
We prove the following theorem in this paper, which extends the corresponding result for $\mathbb{Z}$ actions in [12], and answers a question proposed by Glasner and Megrelishvili in [6].

Theorem 1.1. Let $G$ be an amenable group acting on a dendrite $X$, and suppose that $K$ is a minimal set for the action. Then $(K, G)$ is equicontinuous, and $K$ is either finite or homeomorphic to the Cantor set.

Recently, Shi and Ye have shown that every amenable group action on uniquely arcwise connected continua (without the assumption of local connectedness) must have a minimal set consisting of 1 or 2 points (see [18]). We end this introduction with the following general question:

What results holding for group actions on dendrites can be extended to actions on uniquely arcwise connected continua?

In the following, we assume all the groups appearing in this paper are countable.

\section{Preliminaries}

2.1. Group actions. Let $X$ be a compact metric space, $\operatorname{Homeo}(X)$ its homeomorphism group, and let $G$ be a group. A group homomorphism $\phi: G \rightarrow \operatorname{Homeo}(X)$ is called an action of $G$ on $X$; we also write $(X, G)$ to denote an action of $G$ on $X$. For brevity, we usually write $g x$ or $g(x)$ instead of $\phi(g)(x)$.

The orbit of $x \in X$ under the action of $G$ is the set

$$
G x=\{g x \mid g \in G\} .
$$

For a subset $A \subseteq X$, set $G A=\bigcup_{x \in A} G x$; a set $A$ is said to be $G$-invariant if $G A=A$; finally, a point $x \in X$ is called a fixed point of the action if $G x=\{x\}$. If $A$ is a $G$-invariant closed subset of $X$ and $\overline{G x}=A$ for every $x \in A$ (that is, the orbit of each point is dense), then $A$ is called a minimal set for the action. In this setting every action has a minimal set by Zorn's lemma.

A Borel probability measure $\mu$ on $X$ is called $G$-invariant if $\mu(g(A))=\mu(A)$ for every Borel set $A \subset X$ and every $g \in G$. The following lemma follows directly from the $G$ invariance of the support $\operatorname{supp}(\mu)$ (which is automatic).

Lemma 2.1. If $(X, G)$ is minimal and $\mu$ is a $G$-invariant Borel probability measure on $X$, then $\operatorname{supp}(\mu)=X$.

Lemma 2.2. Suppose that a group $G$ acts on a compact metric space $X$, and that $K$ is a minimal set in $X$ carrying a G-invariant Borel probability measure $\mu$. If $U$ and $V$ are open sets in $X$ such that $V \supset U$ and $g(V \cap K) \subset U \cap K$ for some $g \in G$, then $K \cap(V \backslash \bar{U})=\emptyset$.

Proof. Assume to the contrary that there is some $u \in K \cap(V \backslash \bar{U})$. Then there is an open neighborhood $W \ni u$ with $W \subset V \backslash \bar{U}$. By Lemma 2.1. we have $\mu(W \cap K)>0$. This then implies that $\mu(V \cap K)=\mu(g(V \cap K)) \leq \mu(U \cap K)<\mu(V \cap K)$, a contradiction.

2.2. Amenable groups. Amenability was first introduced by von Neumann. Recall that a countable group $G$ is said to be amenable if there is a sequence of finite sets $F_{i}$ ( $i=$ $1,2,3, \ldots)$ such that

$$
\lim _{i \rightarrow \infty} \frac{\left|g F_{i} \triangle F_{i}\right|}{\left|F_{i}\right|}=0
$$

for every $g \in G$, where $\left|F_{i}\right|$ is the number of elements in $F_{i}$. The sequence $\left(F_{i}\right)$ is called a Følner sequence and each $F_{i}$ a Følner set. It is well known that solvable groups and finite groups are amenable and that any group containing a free non-commutative subgroup is 
not amenable. One may consult the monograph of Paterson [14] for the proofs of the following lemmas.

Lemma 2.3. Every subgroup of an amenable group is amenable.

Lemma 2.4. A group $G$ is amenable if and only if every action of $G$ on a compact metric space $X$ has a G-invariant Borel probability measure on $X$.

2.3. Dendrites. A continuum is a non-empty connected compact metric space. A continuum is said to be non-degenerate if it is not a single point. An arc is a continuum which is homeomorphic to the closed interval $[0,1]$. A continuum $X$ is uniquely arcwise connected if for any two points $x \neq y \in X$ there is a unique arc $[x, y]$ in $X$ connecting $x$ and $y$.

A dendrite $X$ is a locally connected, uniquely arcwise connected, continuum. If $Y$ is a subcontinuum of a dendrite $X$, then $Y$ is called a subdendrite of $X$. For a dendrite $X$ and a point $c \in X$, if $X \backslash\{c\}$ is not connected, then $c$ is called a cut point of $X$; if $X \backslash\{c\}$ has at least 3 components, then $c$ is called a branch point of $X$.

Lemmas 2.5 to 2.8 are taken from [11].

Lemma 2.5. Let $X$ be a dendrite with metric $d$. Then, for every $\varepsilon>0$, there is a $\delta>0$ such that $\operatorname{diam}([x, y])<\varepsilon$ whenever $d(x, y)<\delta$.

Lemma 2.6. Let $X$ be a dendrite. If $A_{i}(i=1,2,3, \ldots)$ is a sequence of mutually disjoint sub-dendrites of $X$, then $\operatorname{diam}\left(A_{i}\right) \rightarrow 0$ as $i \rightarrow \infty$.

Lemma 2.7. Let $X$ be a dendrite. Then $X$ has at most countably many branch points. If $X$ is nondegenerate, then the cut point set of $X$ is uncountable.

Lemma 2.8. Let $X$ be a dendrite and $c \in X$. Then each component $U$ of $X \backslash\{c\}$ is open in $X$, and $\bar{U}=U \cup\{c\}$.

Now we give a proof of the following technical lemma.

Lemma 2.9. Let $X$ be a dendrite and let $f: X \rightarrow X$ be a homeomorphism. Suppose o is a fixed point of $f$, and let $c_{1}, c_{2}$ be cut points of $X$ different from $o$. Suppose that $U$ is a component of $X \backslash\left\{c_{1}\right\}$ not containing $o$, that $V$ is a component of $X \backslash\left\{c_{2}\right\}$ not containing $o$, and that $f\left(c_{1}\right) \in V$. Then $f(U) \subset V$.

Proof. Assume to the contrary that there is some $u \in U$ with $f(u) \notin V$. Since $c_{2}$ is a cut point, $f\left(c_{1}\right) \in V, o \notin V$, and $f(o)=o$, we have $c_{2} \in\left[f(o), f\left(c_{1}\right)\right]$ and $c_{2} \in\left[f(u), f\left(c_{1}\right)\right]$. This implies that $f^{-1}\left(c_{2}\right) \in\left[o, c_{1}\right] \cap\left[u, c_{1}\right]=\left\{c_{1}\right\}$ since $o \notin U$. Thus $f\left(c_{1}\right)=c_{2}$, which contradicts the assumption that $f\left(c_{1}\right) \in V$.

If $[a, b]$ is an arc in a dendrite $X$, denote by $[a, b),(a, b]$, and $(a, b)$ the sets $[a, b] \backslash$ $\{b\},[a, b] \backslash\{a\}$, and $[a, b] \backslash\{a, b\}$, respectively.

2.4. Equicontinuity. Let $X$ be a compact metric space with metric $d$, and let $G$ be a group acting on $X$. Two points $x, y \in X$ are said to be regionally proximal if there are sequences $\left(x_{i}\right),\left(y_{i}\right)$ in $X$ and $\left(g_{i}\right)$ in $G$ such that $x_{i} \rightarrow x$ and $y_{i} \rightarrow y$ as $i \rightarrow \infty$, and $\lim g_{i} x_{i}=$ $\lim g_{i} y_{i}=w$ for some $w \in X$. If $x, y$ are regionally proximal and $x \neq y$, then $\{x, y\}$ is said to be a non-trivial regionally proximal pair. The action $(X, G)$ is equicontinuous if, for every $\varepsilon>0$, there is a $\delta>0$ such that $d(g x, g y)<\varepsilon$ for all $g \in G$ whenever $d(x, y)<\delta$.

The following lemma can be found in [1].

Lemma 2.10. Suppose $(X, G)$ is a group action. Then $(X, G)$ is equicontinuous if and only if it contains no non-trivial regionally proximal pair. 


\section{PROOF OF THE MAIN THEOREM}

In this section we are going to show our main result. Before doing this we state two simple lemmas.

Lemma 3.1. Suppose a group $G$ acts on the closed interval $[0,1]$. If $K \subset[0,1]$ is minimal, then $K$ contains at most 2 points.

Proof. Let $x=\inf K$ and $y=\sup K$. Then $G$ preserves the set $\{x, y\}$, so $K=\{x, y\}$ by the minimality of $K$.

Lemma 3.2 (See [17]). Let $G$ be an amenable group acting on a dendrite $X$. Then there is a G-invariant set consisting of 1 or 2 points.

Now we are ready to prove the main result.

Proof of Theorem 1.1. We first show that $(K, G)$ is equicontinuous.

Assume to the contrary that $(K, G)$ is not equicontinuous. Then by Lemma 2.10 there are $u \neq v \in K$ such that $u, v$ are regionally proximal; that is, there are sequences $\left(u_{i}\right),\left(v_{i}\right)$ in $X$ and $\left(g_{i}\right)$ in $G$ with

$$
u_{i} \rightarrow u, v_{i} \rightarrow v, \lim g_{i} x_{i}=\lim g_{i} y_{i}=w
$$

as $i \rightarrow \infty$ for some $w \in K$.

By Lemma 3.2, there are $o_{1}, o_{2} \in X$ such that $\left\{o_{1}, o_{2}\right\}$ is a $G$-invariant set. Then $\left[o_{1}, o_{2}\right]$ is $G$-invariant by the unique arcwise connectedness of $X$. From the assumption, $K$ is infinite so $K \cap\left[o_{1}, o_{2}\right]=\emptyset$ by Lemma 3.1. Without loss of generality, we may suppose that $o_{1}=o_{2}$ and denote this common point by $o$; otherwise, we need only collapse $\left[o_{1}, o_{2}\right]$ to one point. Then $o$ is a fixed point for the action.

Case 1. $[u, o] \cap[v, o]=\{o\}$ (see Fig.1(1)). By Lemma 2.7, we can choose cut points $c_{1} \in$ $(u, o)$ and $c_{2} \in(v, o)$. Let $D_{u}$ be the component of $X \backslash\left\{c_{1}\right\}$, which contains $u$; let $D_{v}$ be the component of $X \backslash\left\{c_{2}\right\}$, which contains $v$. From minimality and Lemma 2.8, there is some $g^{\prime} \in G$ with $g^{\prime} w \in D_{u}$. From (3.1) and Lemma 2.5, we have

$$
u_{i} \in D_{u}, v_{i} \in D_{v} \text { and } g^{\prime} g_{i}\left[u_{i}, v_{i}\right] \subset D_{u}
$$

for large enough $i$. Write $g=g^{\prime} g_{i}$. Then $o \in\left[u_{i}, v_{i}\right]$ and $g(o) \in D_{u}$. This is a contradiction, since $o$ is fixed by $G$.

Case 2. $[u, o] \cap[v, o]=[z, o]$ for some $z \neq o$.

Subcase 2.1. $z=v$ (see Fig.1(2)). Then $u \neq z$ and $z \in K$. Take a cut point $c_{1} \in(u, z)$ and let $D_{u}$ be the component of $X \backslash\left\{c_{1}\right\}$ which contains $u$. Then $v \notin D_{u}$, and there is some $g \in G$ with $g z \in D_{u}$ by the minimality of $K$. Take a cut point $c_{2} \in(z, o)$ which is sufficiently close to $z$ to ensure that $g\left(c_{2}\right) \in D_{u}$. Let $D_{z}$ be the component of $X \backslash\left\{c_{2}\right\}$ which contains $z$. By Lemma 2.4, there is a $G$-invariant Borel probability measure on $K$. Applying Lemma 2.9. we get $g\left(D_{z}\right) \subset D_{u}$, which contradicts Lemma 2.2, since $z \in D_{z} \backslash \bar{D}_{u}$.

Subcase 2.2. $z=u$. In this case we can deduce a contradiction along the lines of the argument in Subcase 2.1.

Subcase 2.3. $z \neq u$ and $z \neq v$ (see Fig.1(3)). Take a cut point $c_{1} \in(u, z)$. Let $D_{u}$ be the component of $X \backslash\left\{c_{1}\right\}$, which contains $u$. Similar to the argument in Case 1 , there is some $g \in G$ with $g(z) \in D_{u}$. Take a cut point $c_{2} \in(z, o)$ which is sufficiently close to $z$ to ensure that $g\left(c_{2}\right) \in D_{u}$. Let $D_{z}$ be the component of $X \backslash\left\{c_{2}\right\}$, which contains $z$. Then $g\left(D_{z}\right) \subset D_{u}$ by Lemma 2.9. This contradicts Lemma 2.2 since $v \in D_{z} \backslash \bar{D}_{u}$. 
Now we prove that if $K$ is not finite, then $K$ is homeomorphic to the Cantor set. If not, then there is some non-degenerate connected component $Y$ of $K$. Clearly, for any $g, g^{\prime} \in G$, either $g(Y)=g^{\prime}(Y)$ or $g(Y) \cap g^{\prime}(Y)=\emptyset$. This, together with Lemma 2.6 and the equicontinuity of $(K, G)$, implies that the subgroup $H=\{g \in G: g(Y)=Y\}$ has finite index in $G$. It follows that $(Y, H)$ is minimal. This contradicts Lemma 3.2 and Lemma 2.3, since $Y$ is a non-degenerate dendrite.

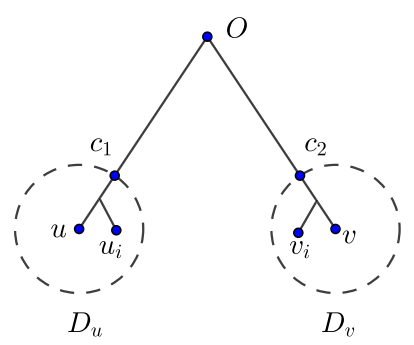

(1)

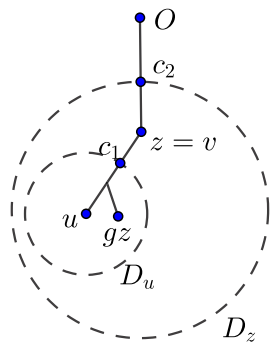

$(2)$

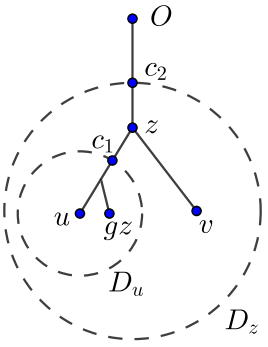

(3)

Fig. 1

Acknowledgements. The authors would like to thank Eli Glasner for sending us the early version of his work with Megrelishvili. The work is supported by NSFC (No. 11771318, 11790274, 11431012).

\section{REFERENCES}

[1] J. Auslander. Minimal flows and their extensions, volume 153 of North-Holland Mathematics Studies. North-Holland Publishing Co., Amsterdam, 1988. Notas de Matemática [Mathematical Notes], 122.

[2] B. H. Bowditch. Hausdorff dimension and dendritic limit sets. Math. Ann., 332(3):667-676, 2005.

[3] B. Duchesne and N. Monod. Group actions on dendrites and curves. Ann. Inst. Fourier, 68(5):2277-2309, 2018.

[4] É. Ghys. Groupes d'homéomorphismes du cercle et cohomologie bornée. In The Lefschetz centennial conference, Part III (Mexico City, 1984), volume 58 of Contemp. Math., pages 81-106. Amer. Math. Soc., Providence, RI, 1987.

[5] É. Ghys. Groups acting on the circle. Enseign. Math. (2), 47(3-4):329-407, 2001.

[6] E. Glasner and M. Megrelishvili. Group actions on treelike compact spaces. Sci. China Math. to appear.

[7] A. V. Malyutin. On groups acting on dendrons. Zap. Nauchn. Sem. S.-Peterburg. Otdel. Mat. Inst. Steklov. (POMI), 415(Geometriya i Topologiya. 12):62-74, 2013. transl. J. Math. Sci. (N.Y.) 212 (2016), 558-565.

[8] G. Margulis. Free subgroups of the homeomorphism group of the circle. C. R. Acad. Sci. Paris Sér. I Math., 331(9):669-674, 2000.

[9] H. Marzougui and I. Naghmouchi. Minimal sets for group actions on dendrites. Proc. Amer. Math. Soc., 144(10):4413-4425, 2016.

[10] Y. N. Minsky. On rigidity, limit sets, and end invariants of hyperbolic 3-manifolds. J. Amer. Math. Soc., 7(3):539-588, 1994.

[11] S. B. Nadler, Jr. Continuum theory, volume 158 of Monographs and Textbooks in Pure and Applied Mathematics. Marcel Dekker, Inc., New York, 1992. An introduction.

[12] I. Naghmouchi. Dynamical properties of monotone dendrite maps. Topology Appl., 159(1):144-149, 2012.

[13] A. Navas. Grupos de difeomorfismos del círculo, volume 13 of Ensaios Matemáticos [Mathematical Surveys]. Sociedade Brasileira de Matemática, Rio de Janeiro, 2007.

[14] A. L. T. Paterson. Amenability, volume 29 of Mathematical Surveys and Monographs. American Mathematical Society, Providence, RI, 1988.

[15] E. Shi. Free subgroups of dendrite homeomorphism group. Topology Appl., 159(10-11):2662-2668, 2012. 
[16] E. Shi, S. Wang, and L. Zhou. Minimal group actions on dendrites. Proc. Amer. Math. Soc., 138(1):217-223, 2010.

[17] E. Shi and X. Ye. Periodic points for amenable group actions on dendrites. Proc. Amer. Math. Soc., 145(1):177-184, 2017.

[18] E. H. Shi and X. Ye. Periodic points for amenable group actions on uniquely arcwise connected continua. arXiv:1710.03411

(E.H. Shi) School of Mathematical Sciences, Soochow University, Suzhou 215006, P. R. CHINA

E-mail address: ehshi@suda.edu.cn

(X. Ye) Wu Wen-Tsun Key laboratory of Mathematics, UStC, Chinese Academy of Sciences and Department of Mathematics, University of Science and Technology of China, HEFEI, ANHUI 230026, CHINA

E-mail address: yexd@ustc.edu.cn 